\title{
PATRONES ESTRUCTURALES EN LAS PLANTAS VASCULARES: UNA REVISION
}

\author{
STRUCTURAL PATTERNS IN VASCULAR PLANTS: A REVIEW
}

\author{
Perreta, M. G. \& A. C. Vegetti \\ Morfología Vegetal, Facultad de Ciencias Agrarias, Universidad Nacional del Litoral, Kreder 2805 (3080) Esperanza, \\ Provincia de Santa Fe, Argentina. mperreta@fca.unl.edu.ar
}

\begin{abstract}
RESUMEN
En las últimas décadas han surgido distintas aproximaciones al aspecto dinámico e integral del crecimiento de las plantas, que interpretan en forma variada su estructura. Estas formas de abordar el estudio de los patrones de crecimiento son: sistema de vástagos, forma de crecimiento, arquitectura vegetal, teoría de plantas clonales y tipología de las inflorescencias. En consecuencia, se ha generado una diversa terminología con distinto grado de precisión y detalle, en ciertos casos equivalente entre enfoques y en otros no. Esta revisión presenta los distintos enfoques y discute las relaciones existentes entre ellos, haciendo énfasis en el estudio del sistema de ramificación de vástagos de la planta.
\end{abstract}

Palabras claves: Plantas clonales, módulo, metámero, sinflorescencia, unidad arquitectural.

\section{ABSTRACT}

The last few decades have seen the development of diverse schools of interpretation of the dynamic growth of plants, namely shoot system analysis, growth form analysis, plant architecture, clonal plant theory and inflorescence typology. This has given rise to a plethora of terminology with diverse degrees of precision and detail. In this review we present these different approaches and discuss the relationships among them, with special emphasis on the study of the branching system of shoots.

KEYwoRDs: Clonal plants, module, metamer, synflorescence, architectural unit.

\section{INTRODUCCION}

La morfología tradicional durante mucho tiempo adoptó un enfoque reduccionista al trabajar sobre órganos aislados más que sobre los aspectos integrales y dinámicos de los sistemas de ramificación (Tomlinson 1987; Bell 1991). Ello estuvo principalmente motivado en que las características estudiadas eran particularidades de órganos aislados, ya que por su gran plasticidad, la forma de la planta fue considerada poco importante. Por otro lado, gran parte de los estudios sobre las respuestas de las plantas al ambiente se expresaron como variaciones en la biomasa de los diferentes órganos, sin prestar atención al comportamiento de las unidades estructurales (Room et al. 1994). Sin embargo, la morfología de desarrollo de la planta en el espacio y en el tiempo tiene implicancias ecológicas y de manejo agronómico muy importantes (Meusel etal. 1977; Montenegro \& Ginocchio 1992), además de posibilitar establecer relaciones taxonómicas y filogenéticas (Meusel 1970; Hagemann 1981).

La morfología vegetal, originada a partir de los trabajos de Goethe en 1790, permanece como relevante para prácticamente todas las disciplinas de la biología vegetal, por el hecho que tales disciplinas refieren o implican conceptos y teorías morfológicas (Sattler \& Rutishauser 1997). No obstante diferentes términos y estructuras conceptuales han ido apareciendo y evolucionando desde ese entonces 
(Kaplan 2001). En las últimas décadas han surgido importantes aportes que hacen al aspecto dinámico e integral del crecimiento de la planta, con un enfoque morfológico o ecológico. Dentro del primero se encuadran las investigaciones realizadas sobre formas de crecimiento (Troll 1964; Meusel 1970; Kästner 1979), y sobre arquitectura vegetal (Hallé \& Oldeman 1970; Hallé et al. 1978); además de trabajos de otros autores como Bell \& Tomlinson (1980), Bell (1986), Barlow (1989) y Troll (1964). Paralelamente a esto, autores como Harper \& White (1974) y Harper (1977) centraron sus estudios ecológicos en la teoría de las plantas clonales. Fruto de estos trabajos se ha generado una cuantiosa terminología que, según el enfoque del que provenga, tendrá niveles de precisión y detalles distintos. Los conocimientos originados por los enfoques ecológicos abarcan siempre un espectro más holístico y con menor detalle descriptivo que los enfoques morfológicos. En estos últimos, las distintas escuelas produjeron terminología específica; muchas veces sin correlación entre ellas y es por este motivo que en la descripción de la estructura y del desarrollo del sistema de ramificación se presentan constantes problemas por el frecuente uso de terminología imprecisa (Bell 1991). Por ello es necesario aclarar la terminología utilizada por las distintas escuelas e intentar explicar su equivalencia.

Este trabajo tiene por objetivo analizar los diferentes enfoques sobre la estructura integral de las plantas, y la terminología específica por ellos generada, como forma de posibilitar una mejor comprensión y aprovechamiento de dicha información.

\section{Sistema DE VÁSTAGOS}

La actividad repetitiva es propia de los procesos morfogénicos. Según Barlow (1989, 1994) esta actividad rítmica resulta de la producción de tres tipos fundamentales de unidades constitutivas. La célula, es la primera, que por su capacidad de autodividirse resulta en el establecimiento del meristema; el funcionamiento y mantenimiento de esta unidad da origen al metámero (segundo tipo de unidad constitutiva). Los metámeros y sus meristemas asociados participan en la construcción del módulo, el tercer tipo de unidad. La combinación de módulos construye un nivel más alto de organización: el sistema de vástagos (Fig. 1 A-C) y el sistema de raíces.
El metámero (también llamado fitómero), unidad elemental de construcción del sistema de vástagos (White 1979, 1984), está compuesto por la hoja, el nudo de inserción, su yema axilar, el entrenudo y en muchos casos raíces adventicias. La variación principal en su definición está dada en el entrenudo que integra el metámero: puede ser el precedente, el siguiente o una porción de él (Bell 1991). También existen diferencias en la posición de la yema axilar y de la hoja (Clark \& Fisher 1986). Sin embargo, lo esencial de esta estructura es su repetitividad más que los detalles de su constitución (White 1979) y el hecho de que cada metámero posee un sitio (yema axilar) con potencialidad de generar un nuevo módulo (Barlow 1989, 1994). En una misma planta puede presentarse una gran variación en la estructura de los metámeros asociada a diferencias en sus partes componentes: forma y longitud del entrenudo, desarrollo o no de yemas axilares, presencia de yemas adventicias, grado de desarrollo de los filomas (desde catafilo a hoja plenamente desarrollada), presencia de raíces adventicias y de modificaciones caulinares o foliares (espinas, zarcillos, aguijones, etc.).

El módulo, según Barlow (1989), es la unidad resultante del desarrollo de una yema. Así considerado es equivalente a un vástago; consecuentemente, es el conjunto de metámeros generados a partir de un meristema apical. De esta forma, el módulo desarrollado a partir de la plúmula y luego de la yema terminal del vástago forma el denominado vástago principal o eje principal de la planta, en tanto que los módulos formados a partir de cada una de las yemas axilares reciben la denominación de vástagos axilares o laterales. La diversidad de módulos observados es consecuencia de variaciones producidas en los metámeros en función de su estructura y su integración. Las características particulares de los módulos (principal o laterales) así como su posición, orientación dentro del sistema de vástagos y tiempo de aparición determina la forma característica de la planta en cada etapa ontogénica, sin descartar las restricciones que las condiciones ambientales pueden generar sobre la misma (Barlow 1994). Estas variaciones en los módulos están dadas por: características de los entrenudos (número y distribución espacial de los distintos tipos), dirección del crecimiento (ortótropos o plagiótropos) y tipos de vástagos asociados a ellos, sucesión foliar, sucesión de distintos tipos de metámeros a lo largo de los 
ejes, momento en que las yemas desarrollan (vástagos silépticos o catalépticos), momento en que las yemas desarrollan inflorescencias o detienen su crecimiento, desarrollo intra o extravaginal de las ramificaciones (de aplicación limitada a algunas monocotiledóneas) y potencialidad de cada metámero de formar un nuevo módulo por ramificación.

La combinación de los tipos de módulos presentes en una planta determina diferentes patrones estructurales y conforma el sistema de vástagos de la planta (Barlow 1994). Este autor considera que el análisis de la estructura vegetal como sistema provee las herramientas necesarias para lograr una representación formal, a través de modelos abstractos, del comportamiento de las plantas.

\section{FORMAS DE CRECIMIENTO}

Se entiende por forma de crecimiento al complejo de caracteres vegetativos y reproductivos genéticamente constantes, que varían sólo dentro de un rango específico de plasticidad fenotípica (Meusel 1952, 1970). El estudio de las formas de crecimiento es comparativo y va unido al análisis de las interacciones con el hábitat, toma en cuenta las etapas sucesivas que llevan a la construcción del cuerpo de la planta desde la germinación (Meusel 1952), y analiza además las distintas fases fenológicas por las que atraviesa durante el año (Meusel etal. 1977; Montenegro \& Ginocchio 1992), principalmente en lo que respecta a exomorfología, aunque puede ser complementado con estudios anatómicos de los distintos órganos. En definitiva, refleja la adaptación local y temporal de la planta a factores abióticos (Meusel 1952, 1970).

El análisis de la forma de crecimiento contempla el estudio detallado de la planta (Fig. 1 D) (Meusel et al. 1977), ya que sólo de la consideración integral de las partes vegetativas y reproductivas se puede tener una idea completa de su estructura (Rua \& Weberling 1998). No considera explícitamente el métamero, pero realiza descripciones cualitativas exhaustivas de sus partes constituyentes a través del análisis de la secuencia foliar, longitud de entrenudos y desarrollo o no de yemas axilares. La más clara definición del sistema de términos que debe utilizarse para describir y clasificar las formas de crecimiento, se encuentra en los trabajos de Mühlberg (1967), quien logra, sin sobredimensionar los aspectos considerados, una completa descripción de la planta. Según este autor y Meusel et al. (1977) es indispensable determinar, entre otras cosas, duración de las estructuras, posición de la ramificación, características del sistema radical y forma de vida. Esta información puede ser complementada con estudios morfométricos (Meusel et al. 1977).

\section{Arquitectura vegetal}

Este enfoque surgió en la década del '70 a partir de los trabajos de Hallé y Oldeman, los que basándose en el estudio de los sistemas de ramificación de árboles tropicales encontraron la relación entre arquitectura y crecimiento, y desarrollaron el marco conceptual para la descripción y clasificación de las plantas (Tourn et al. 1999).

La arquitectura de una planta es el resultado del funcionamiento de sus meristemas (Tourn et al. 1999), queda determinada por el número, tamaño y disposición relativa de sus ejes vegetativos aéreos y subterráneos (Hallé \& Oldeman 1970), y por la reorientación activa que estos ejes puedan sufrir en el medio en el cual se desarrollan (Bell 1994). Es la expresión de un equilibrio entre el programa de desarrollo endógeno y las acciones ejercidas por el ambiente (Edelin 1984). El objetivo del análisis arquitectural es identificar esta secuencia endógena de desarrollo y diferenciarla de los efectos debidos a la influencia del medio (Puntieri et al. 1995). Para ello es necesario lograr la síntesis del patrón de desarrollo a partir de la observación de las características morfológicas de sus unidades estructurales y de la determinación de la secuencia de diferenciación ontogénica de éstas (Puntieri et al. 1995). Este enfoque morfológico es dinámico y global (Blaise et al. 1998) y abarca desde la germinación hasta la muerte del individuo (Edelin 1984).

La unidad de construcción de la planta que repetidamente ha probado tener valor en la elucidación de su estructura es el meristema apical y la estructura que él desarrolla: el eje foliado (Bell 1994). A partir de esta unidad formada por subunidades menores (metámeros) se basa el estudio de la arquitectura vegetal (Barthélémy 1991), ya que un sistema ramificado puede ser descrito en términos de jerarquía de unidades de orden sucesivo (Bell 1993). Esto involucra la repetición e integración de varios niveles de organización como ser el metámero (Fig. 1E), la unidad de crecimiento, la unidad simpodial, el vástago anual, el eje foliado (Fig. 1 F) y la unidad arquitectural (Fig. 1G) (Barthélémy 1991). 
Existen tres conceptos básicos y fundamentales para la arquitectura vegetal, ellos son: modelo arquitectural, unidad arquitectural y reiteración. El modelo arquitectural representa la estrategia de crecimiento global de la planta (Tourn et al. 1999). Se define como la serie de estructuras espaciales (arquitecturas) formadas por la planta durante el crecimiento en un ambiente no traumático (Blaise et al. 1998) y es fundamentalmente un concepto dinámico que se representa a través de una secuencia de esquemas (Tourn et al. 1999). El modelo resulta de la combinación de diferentes tipos de ejes con características morfológicas básicas de fácil observación a campo, como son: tipo de crecimiento, tipo de ramificación, distribución de las ramas en la entidad portadora, filotaxis, orientación de los ejes en el espacio, y la presencia o ausencia y posición de las estructuras sexuales (Barthélémy 1991). Si bien el número de combinaciones teóricas es alto, sólo existen alrededor de 23 modelos en la naturaleza, que se aplican indistintamente a especies herbáceas y arbóreas, tanto tropicales como templadas, y a taxones actuales o extintos (Tourn et al. 1999). Los modelos no son más que los programas de crecimiento más frecuentes adoptados dentro de un continuum arquitectural (Tourn et al. 1999). Los patrones de crecimiento definidos por estos modelos están determinados genéticamente, su expresión sólo es afectada ante condiciones ecológicas extremas (Barthélémy et al. 1995). Esta clasificación posee fundamentalmente un interés teórico (Barthélémy 1991).

La unidad arquitectural caracteriza la arquitectura elemental de la especie y permite describir completa y precisamente una planta; es la expresión específica del modelo (Barthélémy 1991). A medida que la planta se desarrolla se establecen jerarquías entre los distintos ejes producidos, donde cada una es identificable por sus características propias. La descripción de todas las categorías de ejes, la forma en que se derivan unas de otras y su disposición relativa al momento de la aparición de las estructuras reproductivas es la unidad arquitectural (Barthélémy \& Caraglio, 1991). Es por esto que su identificación necesita de una completa diagnosis de los aspectos morfológicos y funcionales de todas las categorías de ejes que se pueden reconocer en cada uno de los estadios de crecimiento (Tourn et al. 1999). La arquitectura elemental se mantiene estable en sus aspectos cualitativos en todos los ambientes donde se desarrolla la especie. En otras palabras, el modelo correspondería al modo de crecimiento; mientras que la unidad arquitectural es el detalle de todos los tipos de ejes presentes.

Luego de la expresión de su unidad arquitectural, el crecimiento ulterior de la mayoría de las plantas se realiza a través de la formación de tipos de ejes ya representados en alguna etapa previa del desarrollo (Puntieri et al. 1995). Esta repetición parcial o total de la arquitectura elemental (unidad arquitectural) durante la ontogenia se conoce con el nombre de reiteración y es el mecanismo esencial por el cual se construye la copa en la mayoría de los árboles (Barthélémy 1991); este proceso también puede producirse en respuesta a traumatismos o a situaciones ambientales cambiantes (Tourn et al. 1999). El resultado de este proceso se denomina complejo reiterado (Fig. $1 \mathrm{H}$ ) y provoca una modificación importante de la arquitectura base. Cada especie expresa su propia estrategia reiterativa de acuerdo a su arquitectura elemental (Barthélémy 1991). Algunas especies, sobre todo anuales, carecen de este proceso siendo la planta equivalente a la unidad arquitectural y no a un complejo reiterado. El análisis arquitectural permite una verdadera cartografía de la estructura vegetal, y prepara el terreno para un análisis más profundo de tipo cuantitativo (Tourn et al. 1999); el que permite la medición y modelización del crecimiento en función de las unidades estructurales que conforman la estructura del vegetal (Godin et al. 1997; Blaise et al. 1998). Estos modelos de crecimiento describen el funcionamiento de los meristemas a través de procesos estocásticos (De Reffye et al. 1991), de esta forma contemplan las variaciones intra e interindividuales debidas a procesos aleatorios, lo cual les permite simular la variabilidad de las poblaciones naturales (Puntieri et al. 1995). Se logra una simulación de la dinámica poblacional, ya que cada módulo dentro de la población tiene su propia edad, o estado, una probabilidad específica de sobrevivir o reproducirse, y una distribución estadística propia para cada parámetro de crecimiento (Callaghan et al. 1990).

\section{LA CONCEPCIÓN MODULAR Y LAS PLANTAS CLONALES}

En las últimas dos décadas hubo una creciente consideración sobre aspectos ecológicos, fisiológicos, genéticos y evolutivos de las plantas 
clonales (Herben et al. 1994; De Kroon \& Van Groenendael 1997). Esto condujo al desarrollo de un enfoque interdisciplinario que involucra biología de poblaciones, ecofisiología, morfología y ecología de comunidades y la posibilidad de abordar el estudio de un vegetal desde el punto de vista demográfico (Briske 1991; Moore \& Moser 1995). Las ideas de organización modular, crecimiento modular y modularidad fueron introducidas por zoólogos y botánicos para describir estrategias de vida en colonias de invertebrados y organismos vegetales pluricelulares y se basan en la morfogénesis repetitiva, no en peculiaridades de las unidades que constituyen el organismo (Marfenin 1999). Esta concepción fue luego aplicada a las plantas superiores, ya que las mismas son también organismos modulares; están construidas por un número potencialmente ilimitado de partes similares (Harper 1977).

Las plantas se desarrollan a partir del cigoto por un proceso de iteración secuencial de unidades de construcción o subunidades (Harper \& White 1974) y su forma o estructura es determinada por la producción y pérdida de estas partes que se repiten (Room et al. 1994). A este conjunto de partes desarrolladas a partir del cigoto, producto de la germinación y posterior crecimiento, se lo denomina genet (Fig.1I) (Harper 1977) desde el punto de vista de la biología de poblaciones metapoblación (Harper $\&$ White 1974).

Por otro parte todo vástago o conjunto de vástagos derivados por multiplicación vegetativa del genet se denomina ramet y es considerado la unidad de crecimiento clonal (Fig.1I), capaz de una existencia independiente de la planta madre (Harper 1977); pese a ello, desde el punto de vista morfológico no ha sido estrictamente definido (White 1979). Los ramets pueden estar próximos a la planta madre o alejados de ella conectados por estolones, rizomas o raíces gemíferas. Estas estructuras morfológicas separadoras de ramets fueron denominadas espaciadores (spacers) (Fig. 1I) (Bell 1986).

La estructura modular tiene implicancias en la historia vital de las especies clonales. La dinámica de módulos y metámeros determina la habilidad de capturar recursos, la productividad y el fitness (Room et al. 1994; Kull 1995). La forma en que los ramets están fisiológicamente integrados varía marcadamente entre especies, y muchas características de la población y de la dinámica de la comunidad dependen del grado de integración clonal dentro del genet (Eckert 1999). La habilidad para moverse por espaciadores a sitios vecinos las hace capaces de mostrar una respuesta más flexible a su ambiente local, escapar de sitios desfavorables y explorar otros ambientes (Herben $e t$ al. 1994). El crecimiento de las plantas clonales conduce a la formación de estructuras espaciales jerárquicas a través de un determinado patrón de ramificación (Bell 1986; Hutchings \& Mogie 1990). Tiffney \& Niklas (1985) y Klimes et al. (1997) proponen diferentes características necesarias para un análisis detallado de las planta clonales como ser: origen del órgano de crecimiento clonal, posición de los ápices que producen ramets, dirección de crecimiento, posición resultante de dichos ramets con respecto a la superficie del suelo, engrosamiento de los órganos del crecimiento clonal para formar estructuras de reserva, longitud del crecimiento radical (para las raíces gemíferas), duración del ramet (duración del meristema apical), características de los espaciadores, frecuencia de ramificación (indicador de la intensidad de la reproducción vegetativa), ángulo de ramificación, densidad de ramets, frecuencia relativa de tipos de ramets, tamaño del ramet (altura del vástago y peso del vástago) .

\section{TIPOLOGÍA DE LAS INFLORESCENCIAS}

Este enfoque presenta una terminología rica y detallada para el estudio de las inflorescencias (Troll 1964; Weberling 1989; Rua 1999) y permite realizar comparaciones entre estructuras homólogas. Su análisis como método para determinar el patrón estructural de las plantas se debe al hecho de que desde el punto de vista tipológico, la inflorescencia, tal como es de uso corriente, correspondería al antotagma (Mora Osejo 1987) o a la unidad de floración (Sell \& Cremmers 1994); y su estudio debe realizarse integrado al plan estructural de toda la planta a través del concepto más amplio de sinflorescencia (Troll 1964; Vegetti \& Anton 1995). Esta última se define como el sistema de vástagos floríferos producidos a partir de la yema apical del eje embrional o de una yema axilar (producción de innovaciones) en los años subsiguientes (Troll \& Weberling 1989), y es la unidad de estudio de este enfoque (Fig. 1J). Es decir que una planta anual posee una sola sinflorescencia y una perenne posee varias (Fig. 1K), según sea su forma de crecimiento (Rua 1999). A nivel del estudio de las estructuras reproductivas, sin importar el hecho de que el eje principal remate en una flor (inflorescencia 

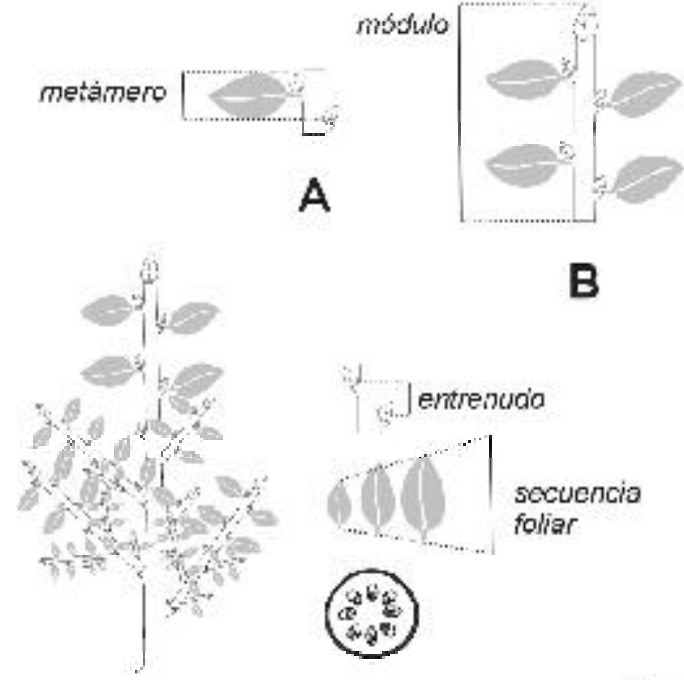

B
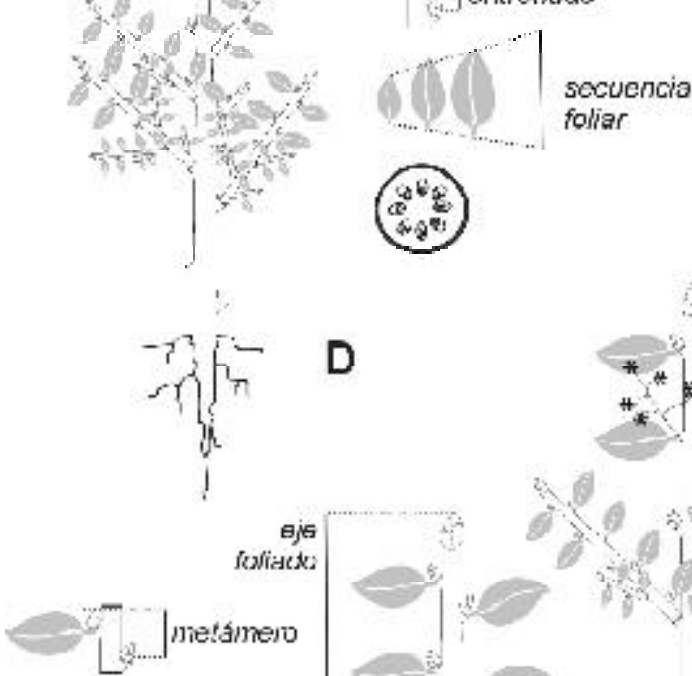

E

$\mathrm{F}$

G

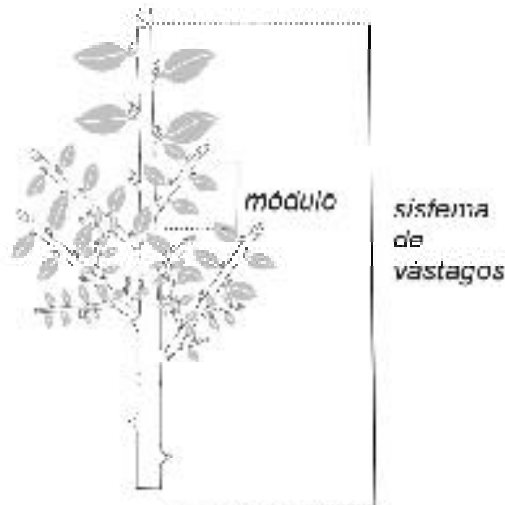

C

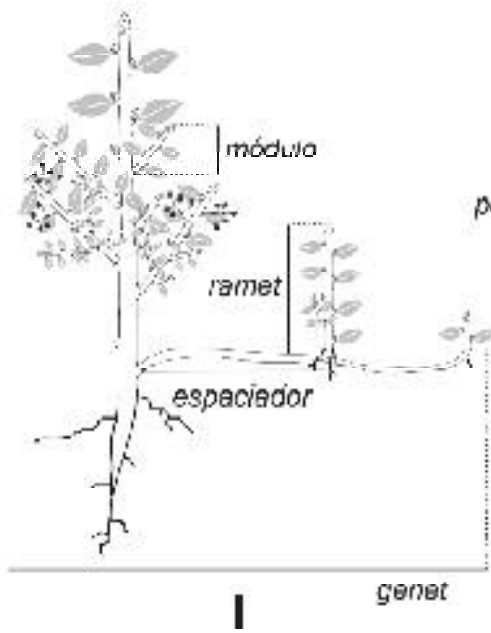

H
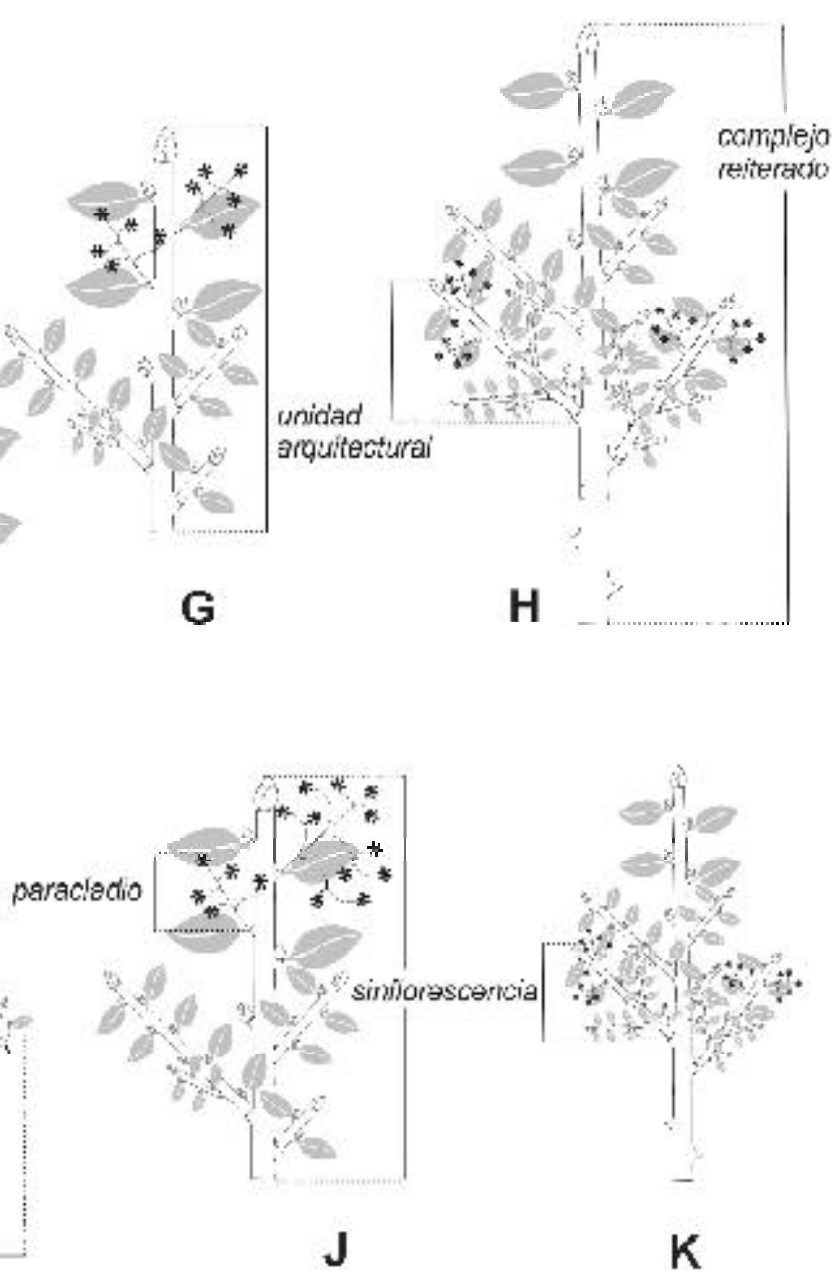

Figura 1: Patrones estructurales en plantas. A-C: sistema de vástagos; D, forma de crecimiento; E-H, arquitectura vegetal; I, plantas clonales; J-K, tipología de las inflorescencias. 
monotélica) o un grupo de flores (inflorescencia politélica), todas las ramificaciones laterales portadoras de flores reciben el nombre de paracladios (Troll 1964; Weberling 1985).

El estudio de la tipología de las inflorescencias surge a partir de los trabajos de Troll (1964), quien desarrolló un marco conceptual al integrar el sistema reproductivo dentro del plan estructural de la planta, y que se basó fundamentalmente en el principio de las proporciones variables. De acuerdo a este principio, el grado de desarrollo alcanzado por determinados elementos constitutivos es responsable de la estabilización de un gran número de formas derivadas.

Actualmente el análisis de las sinflorescencias incorpora estudios de la dinámica de desarrollo para generar modelos que describen las inflorescencias relacionando estructuras adultas, mecanismos genéticos y transformaciones que ocurren durante la ontogenia (Kellog 2000). La complementación entre el análisis tipológico y de desarrollo de las inflorescencias contribuye a una mejor interpretación de la complejidad presente en los sistemas de ramificación de los vástagos floríferos (Weber \& Vegetti 2001; Reinheimer et al. 2005).

\section{RELACIONENTRE LOS DISTINTOSENFOQUES}

Para reconocer y describir la secuencia de ramificación de una planta es útil identificar sus unidades de construcción y la manera en que ellas están integradas (Bell 1991), ya que una estructura compleja puede ser mejor comprendida si se descompone en elementos más simples, que pueden así ser medidos y analizados matemáticamente (Bell 1993). La disposición espacial de los módulos determina la estructura de la planta y la secuencia de formación temporal, durante la ontogenia y las fases fenológicas, de cada uno de ellos magnifica la variabilidad que puede ser observada en la construcción modular (Montenegro \& Ginocchio 1992). Todos los enfoques expresan el crecimiento de la planta como repetición de subunidades, aunque divergen en el nivel de precisión y detalle de estas unidades repetitivas (Tabla I) y, salvo la tipología de las inflorescencias, han adaptado el sistema que utilizan en la descripción de la estructura de la parte aérea, para describir el sistema radical (Barlow 1989; 1994; Lynch 1995; Klimes et al. 1997; Danjon et al. 1999). Del mismo modo todos los enfoques han sido aplicados a distintas formas vegetales desde árboles hasta hierbas (Jeanoda-Robinson 1977; Kästner 1979; Barthélémy et al. 1991; Bell 1993; Rua \& Weberling 1998; Perreta 2004).

Tanto el sistema de vástagos proveniente de la germinación de la semilla y posterior crecimiento (genet) como las partes vegetativas que lo integran (ramets y espaciadores) construyen la estructura de la planta. Los ramets pueden conformar o no un módulo (Fig. 2), lo cual no inhabilita el hecho de que puedan a su vez portar o no módulos laterales. En este trabajo se considera al módulo según Barlow (1989), es decir equivalente a un vástago (la producción de un meristema apical) o a un eje foliado (Bell 1994). Así aplicado, una rama monopodial es una unidad de vástago o un módulo (Fig. 2 A); y una rama simpodial es una serie de unidades de vástago (o de módulos), cada una derivada de un único meristema apical (Fig. 2 B).

TABLA I. Comparación de los niveles de organización de las estructuras del vástago para los enfoques analizados.

\begin{tabular}{|c|c|c|c|c|}
\hline & Unichd elemental & $\begin{array}{l}\text { Segundo nirel de } \\
\text { camplejilad }\end{array}$ & $\begin{array}{l}\text { Tercer rimel de } \\
\text { comple jind }\end{array}$ & $\begin{array}{c}\text { Cuarto rimel de } \\
\text { complejudad }\end{array}$ \\
\hline $\begin{array}{l}\text { Sistema de vísthos } \\
\text { Forma de aecin iento }\end{array}$ & $\begin{array}{l}\text { Cébula } \\
\text { Planth }\end{array}$ & Mfetím ero & Módulo & Sistema de váthgos \\
\hline $\begin{array}{l}\text { inquitecturn } \\
\text { Clanales }\end{array}$ & $\begin{array}{l}\text { Metín ero } \\
\text { Metím ero }\end{array}$ & $\begin{array}{l}\text { Itótub "I) } \\
\text { Mótulb }\end{array}$ & $\begin{array}{c}\text { Unidid arquitectornl } \\
\text { Ramet. }\end{array}$ & $\begin{array}{c}\text { Complejoretendo } \\
\text { Geret. }\end{array}$ \\
\hline Tyologin inflare scerc ins & $\begin{array}{c}\text { Fla o grupo de flares } \\
\text { (flla exenci) }\end{array}$ & Paracladio & Sinflorescencin & Plonth \\
\hline
\end{tabular}


Sin embargo, el concepto módulo ha sido aplicado con otras acepciones: como traducción del término francés l'article (Hallé et al. 1978); o de forma vaga para significar una unidad de construcción que es repetida cuando la planta desarrolla. Según esta última forma, el módulo puede ser una hoja, un metámero, un artejo, una unidad del sistema de ramificación o un ramet (Bell 1991). El término l'article, por otra parte se refiere a una unidad simpodial, es decir cada unidad de una rama simpodial (Bell 1991), y es equivalente al término caulómero (Stone 1975).

Los ramets pueden estar cerca de la planta madre o alejados; ello depende del número y longitud de los entrenudos (para los estolones y rizomas) y de la longitud del crecimiento radical (para las raíces gemíferas). Así entonces un espaciador (si es un vástago) puede constar de uno o más entrenudos de longitud variable. Un ramet constituye un módulo cuando es producto de una yema axilar de un vástago de crecimiento plagiótropo monopodial (Fig. 2 A). Por otro lado, cuando el ramet se desarrolla como consecuencia del crecimiento simpodial del eje plagiótropo, sólo constituye una parte del módulo, ya que el mismo, al ser el producto del desarrollo de una yema estaría constituido por el espaciador más el eje ortótropo que forma el ramet (Fig. 2B).

Es posible encontrar niveles de integración intermedios entre metámero y módulo, fundamentalmen- te en árboles de clima templado, denominados unidades de crecimiento, sucesión de metámeros producidos durante un período de alargamiento ininterrumpido (Barthélémy 1991), o submódulos, secuencias de metámeros morfológicamente diferentes (Barlow 1994).

Un conjunto de vástagos conforma un sistema de vástagos, el arreglo espacial de los mismos determina la morfología particular de la planta y corresponde a uno de los modelos arquitecturales definidos por la escuela francesa (Barlow 1994). Sin embargo, la definición del modelo arquitectural es muy general y no permite describir las peculiaridades del sistema de ramificación de cada especie, lo que sí es posible a través de la unidad arquitectural, que consiste en el conjunto de diferentes tipos de módulos formados en la planta hasta la primera floración (Barthélémy \& Caraglio 1991). Una planta anual constituye una unidad arquitectural (Tourn et al. 1999), es decir que en este caso la unidad arquitectural sería equivalente a la sinflorescencia. Por otro lado los paracladios, término utilizado en las descripciones tipológicas para referirse a ramificaciones portadoras de flores, no poseen correlación dentro de los demás sistemas descriptivos, dado que no se aplican al análisis detallado de las estructuras reproductivas. Estos paracladios, al ser el producto de una yema pueden ser considerados equivalentes a un módulo.

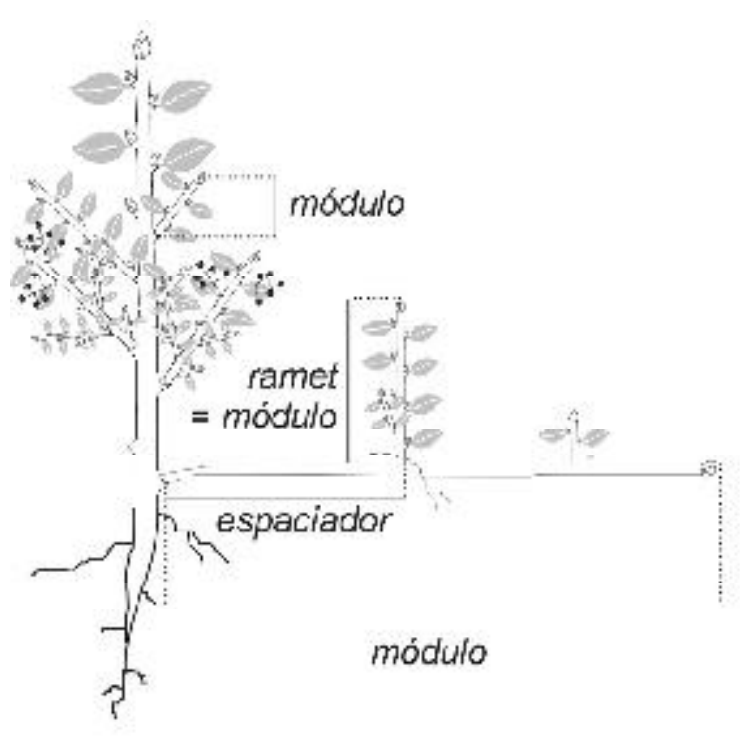

A

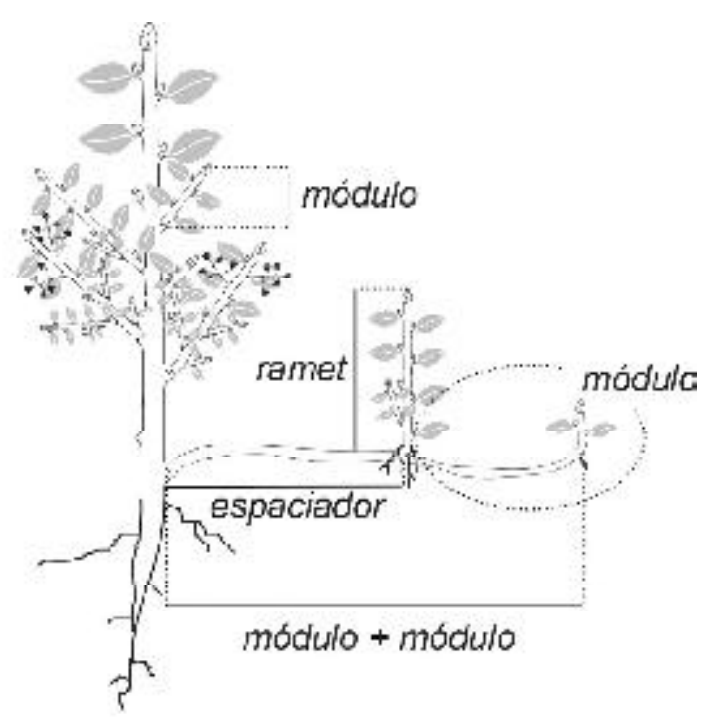

B

FIGURA 2. Relación entre los términos módulo (según Barlow 1989) y ramet-espaciador. A: en una rama plagiótropa monopodial; B: en una rama plagiótropa simpodial. 
A lo largo de las distintas etapas ontogénicas y fenológicas se generan diversos tipos de módulos que se diferencian en sus funciones (Montenegro \& Ginocchio 1992). La partición de funciones en unidades arquitecturales básicas da más plasticidad a la planta, y la proporción de módulos asignados a diferentes roles está estrechamente relacionada con el ambiente (Barthélémy 1986). Las diferencias encontradas en la distribución de los diversos módulos entre diferentes formas de crecimiento puede relacionarse con el tipo de ambiente donde cada una de ellas predomina (Montenegro \& Ginocchio 1992).

La planta se va construyendo por acumulación jerárquica de unidades de vástago (módulos) producidos por un meristema apical que puede tener crecimiento indefinido (monopodial) o definido (simpodial). Lo importante en este tipo de estudios es reconocer la naturaleza dinámica de los sistemas de ramificación, y la particularidad de cada especie de presentar una estructura singular de su sistema de ramificación, como consecuencia de un arreglo particular de unidades de vástago, en el espacio y en el tiempo. Este concepto de construcción fitomérica ha demostrado ser particularmente útil en la interpretación de la estructura y plasticidad morfológica de numerosas especies (Clark \& Fisher 1986).

\section{BIBLIOGRAFIA}

BARLOW, P. W. 1989. Meristems, metamers and modules and the development of shoot and root systems. Botanical Journal of the Linnean Society 100: 255-279.

BarLow, P. W. 1994. From cell to system: repetitive units of growth in the development of roots and shoots. In: Growth Patterns in Vascular Plants. (ed. M. Iqbal), pp. 19-58. Dioscorides Press, Portland.

BARTHÉLÉMY, D. 1986. Establishment of modular grouwth in a tropical tree: Isertia coccinea Vahl. (Rubiaceae). Philosophical Transactions of the Royal Society of London, Series B 313: 89-94.

BARTHÉLÉmY, D. 1991. Levels of organization and repetition phenomena in seed plants. Acta Biotheoretica 39: 309-323.

Barthélémy, D., C. Edelin \& F. Halle. 1991. Canopy Architecture. In: Physiology of trees (ed A.S. Raghavendra ), pp. 1-19. J. Willey \& Sons, Inc. London.

Barthélémy, D., F. Blaise, T. Fourcaud \& E. Nicolini. 1995. Modélisation et simulation de l'architecture des arbres: bilan et perspectives. Revue Forestiere de France 47: 71-96.
Barthélémy, D. \& Y. Caraglio. 1991. Modélisation et simulation de l'architecture des arbres. Forêtentrerprise 73: 28-39.

BELL, A. 1986. The simulation of branching patterns in modular organisms. Philosophical Transactions of the Royal Society of London, Series B 313: 143-159.

BELL, A. 1991. Plant form. An illustrated guide to flowering plant morphology. Oxford University Press, Hong Kong. 341 pp.

BELL, A. 1993. Les plantes à fleur: Guide morphologique illustré. Masson, Paris. 341 pp.

BELL, A. 1994. A summary of branching process in plants. In: Shape and form in plants and fungi (eds. D.S. Ingram \& A. Hudson), pp. 119-142. Academic Press, London.

Bell, A. \& P.B. Tombinson. 1980. Adaptive architecture in rhizomatous plants. Botanical Journal of the Linnean Society 80: 125-160.

Blaise, F., J.F. Barczi, M. Jaeger, P. Dinouard \& P. DE REFFYE. 1998. Simulation of the growth of plants. Modelling of metamorphosis and spatial interactions in architecture and development of plants. In: Cyberworlds (eds. T.L. Kunii \& A. Luciani), pp. 81-109. John Wiley \& Sons, Tokyo.

BRISKE, D.D. 1991. Developmental Morphology and Physiology of Grasses. In: Grazing management: An ecological perspective (eds. R. K. Heitschmidt and J. W. Stuth), pp. 85-108. Timber Press, Portland.

Callaghan, T.V., B.M. Suensson, H. Bowman, D.K. LINDLEY \& B.A. CARLSSON. 1990. Models of clonal plant growth based on population dynamics and architecture. Oikos 57: 257-269.

Clark, L. \& J. Fisher. 1986. Vegetative Morphology of grasses: shoots and roots. In: Grass systematics and evolution. (eds. T.R. Soderstrom, K.W. Hilu, C.S. Campbell, \& M.E. Barkworth), pp. 37-47. Smithsonian, Washington DC.

Danjon, F., D. Bert, C. Godin \& P. Trichet. 1999. Structural root architecture of 5-years-old Pinus pinaster measured by $3 \mathrm{D}$ digitising and analysed with AMAPmod. Plant and Soil 217: 49-63.

ECKER T, C.G. 1999. Clonal plant research: proliferation, integration, but not much evolution. American Journal of Botany 86: 1649-1654.

EDELIN, C. 1984. L'architecture monopodiale: l' exemple de quelques arbres d'Asie tropicale. PhD Thése. Universite de Montpellier II, France. 258 pp.

Godin, C., Y. Guédon, E. Costes \& Y. Caraglio. 1997. Measuring and analyzing plants with AMAPmod software. In: Plants to ecosystems. (ed. M.T. Michalewicz.), pp. 53-84. CSIRO, Australia.

Hagemann, W. 1981. Wuchsformenuntersuchungen an balkanischen Hypericum-sippen. Botanische Jahrbücher für Systematik, Pflanzengeschichte und Pflanzengeographie 102: 239-254.

Hallé, F. \& R. A. Oldeman. 1970. Essai sur l'architecture et la dynamique de croissance des arbres tropicaux. Masson. Paris. 198 pp. 
Hallé, F., R. A. Oldeman \& P.B. Tomlinson. 1978. Tropical trees and forests. Springer Verlag, Berlín. 442 pp.

HarPer, J. L. 1977. Population biology of plants. London Academic Press, London. 892 pp.

Harper, J. L. \& J. White. 1974. The demography of plants. American Review of Ecology and Systematics 5: 419-463.

Herben, T., T. Hara, Ch. Marshall \& L. Soukupová. 1994. Plant clonality: Biology and Diversity. Folia Geobotanica \& Phytotaxonomica 29: 113-122.

Hutchings, M. J. \& M. Mogie. 1990. The spatial structure of clonal plants: control and consequences In: Clonal growth in plants: regulation and function. (eds. J. M. van Groenendael \& H. de Kroon.), pp. 57-76. SPB Academic Publishing, The Hague.

Jeannoda-Robinson, V. 1977. Contribution a l'etude de l'architecture des herbes. PhD Thése. Université des Sciences et Techniques du LanguendocMontpellier, France. 262 pp.

KAPLAN, D.R. 2001. The science of plant morphology: definition, history, and role in modern biology. American Journal of Botany 88: 1711-1741.

KästNER, A. 1979. Beiträge zur Wuchsformanalyse und systematischen Gleiderung von Teucrium L. II. Anatomie der Sprosse und Blätter. Flora 168: 431-467.

Kellog, E. 2000. A model of inflorescence development. In: Monocots, Systematics and Evolution, vol. 1 (eds W.L. Jacobs \& J. Everett), pp. 84-88. Csiro Publishing, Melbourne.

Klimes, L., J. Klimesova, R. Hendriks \& J. van Groenendael. 1997. Clonal plant architecture: a comparative analysis of form and function. In: The ecology and evolution of clonal plants (eds H. de Kroon \& J. van Groenendael), pp. 1-29. Backhuys Publishers, Leiden.

Kroon, H. de \& J. van Groenendael (eds.). 1997. The ecology and evolution of clonal plants. Backhuys Publishers, Leiden. pp. 453.

KuLL, K. 1995. Growth form parameters of clonal herbs. In: Consortium masingii: A festschrift for Viktor Masing (eds. Aaviksook, K., K. Kull, J. Pall \& H. Trass). Scripta Botanica 9: 106-115. URL: http:/ /www.zbi.ee/ kalevi/growth.htm: Viewed: February 22, 2002.

LYNCH, J. 1995. Root architecture and plant productivity. Plant Physiology 109: 7-13.

M ARFENIN, N.N. 1999. The development of modular organization concept. Journal of General Biology 60: 6-17.

Meusel, H. 1952. Über Wuchsformen, Verbreitung und Phylogenie einiger mediterran-mitteleuropäischen Angiospermen-Gattungen. Flora 139: 333-393.

Meusel, H. 1970. Wuchsformenreihen Mediterrananmitteleuropäischer Angiosperm-Taxa. Feddes Repertorium 81: 41-59.

Meusel, H., E. JäGer \& G. M ÖRChEn. 1977. The study of growth-forms of higher plants in relation to application of biocide. Vegetation Science and Environmental Protection: 71-76.

Montenegro, G. \& R. Ginocchio. 1992. Modular interpretation of architecture in shrub species. Anais da Academia Brasileira de Ciencias 65: 189-202.

Moore, K. J. \& L. E. Mooser. 1995. Quantifying developmental morphology of perennial grasses. Crop Science 35: 37-43.

M ora Osejo, J. E. 1987. Estudios Morfológicos, Autoecológicos y Sistemáticos en Angiospermas. Academia Colombiana de Ciencias Exactas, Físicas y Naturales, Bogotá. 253-341 pp.

Mühlberg, H. 1967. Die Wuchstypen der mitteldeutschen Poaceen. Hercynia 4: 11-50.

Perreta, M.G. 2004. Caracterización de los patrones de desarrollo en Melica L. (Meliceae - POACEAE): análisis arquitectural. $\mathrm{PhD}$ Tesis. Facultad de Bioquímica y Ciencias Biológicas-Universidad Nacional del Litoral, Argentina. 185 pp.

Puntieri, J, D. Barthélémy, P. De Reffye \& C. Brion. 1995. Análisis, modelización y simulación de la arquitectura de las plantas. In: Avances y aplicaciones en la región Andino-Patagónica, pp. 547-573. Actas de las IV Jornadas Forestales Patagónicas, Tomo II, San Martín de los Andes.

Reffye, P. DE, E. Elguero \& E. Costes. 1991. Growth units construction in trees: a stochastic approach. Acta Biotheoretica 39: 325-342.

Reinheimer, R., R. Pozner \& A. C. Vegetti. 2005. Inflorescence, spikelet and floral development in Panicum maximum and Urochloa plantaginea (Poaceae). American Journal of Botany 92: 565-575.

Room, P.M., L. Maillette \& J.S. Hanan. 1994. M odule, metamer dynamics and virtual plants. In: Advances in Ecological Research 25 (eds. M. Begon \& A.H. Fitter), pp. 105-157. Academic Press, London.

RuA, G. 1999. Inflorescencias. Bases teóricas para su análisis. Sociedad Argentina de Botánica, Buenos Aires. 100 pp.

Rua, G. \& F. Weberling. 1998. Growth form and inflorescence structure of Paspalum L. (Poaceae: Paniceae): a comparative morphological approach. Beiträge zur Biologie der Pflanzen 69: 363-431.

Sell, Y. \& G. CRemmers. 1994. Identification de l'unité de floraison des Marantacées. Beiträge zur Biologie der Pflanzen 68: 51-59.

Stone, B.C. 1975. Authorized translation of "An essay on the architecture and dynamics of growth of tropical trees" (eds. F. Hallé \& R. A. A. Oldeman). Penerbit University, Malaya, Kuala Lumpur. 200 pp.

Sattler, R. \& R. Rutishauser. 1997. The fundamental relevance of morphology and morphogenesis to plant research. Annals of Botany 80: 571-582.

Tiffney, B. H. \& K. J. NikLas. 1985. Clonal growth in land plants: a paleobotanical perspective. In: Population biology and evolution of clonal organisms (eds. J. Jackson, L. Buss \& R. Cook). pp. 
35-65. Yale University Press, New Haven.

Tomlinson, P. B. 1987. Branching is a process, not a concept. Taxon 36: 54-57.

Tourn, G. M., D. B ARThéLÉmy \& J. Grosfeld. 1999. Una aproximación a la arquitectura vegetal: conceptos, objetivos y metodología. Boletín de la Sociedad Argentina de Botánica 34: 85-99.

Troll, W. 1964. Die Infloreszenzen, Typologie und Stellung im Aufbau des Vegetationskörpers 1. Gustav Fischer, Jena.

Troll, W. \& F. Weberling. 1989. Infloreszenzuntersuchungen an monotelen Familien Materialen zur Infloreszenzmorphologie. G. Fischer, Stuttgart. 490 pp.

Vegetti, A.C. \& A.M. Anton. 1995. Some evolution trends in the inflorescence of Poaceae. Flora 190: 225-228.
Weber, M. \& A. C. Vegetti. 2001. An analysis of the inflorescences in species of Luziola (Oryzeae Poaceae). Beiträge zur Biologie der Pflanzen 72: 161-179.

WeberLing, F. 1985. Aspectos modernos de la morfología de las inflorescencias. Boletín de la Sociedad Argentina de Botánica 24: 1-28.

Weberling, F. 1989. Morphology of flowers and inflorescences. Trans. by J. Pankhurst. Cambridge University Press, Cambridge. 405 pp.

White, J. 1979. The plant as a metapopulation. Annual Review of Ecology and Systematics 10: 109-145.

White, J. 1984. Plant metamerism. In: Perspectives on plant population ecology (eds. Dirzo, R. \& J. Sarukán.), pp. 15-47. Sinauer Associates, Masssachusetts. 